\title{
Automated geolocalised identification of polyhedral blocks and their safety factor calculation in open pit mining
}

\author{
F González Antofagasta Minerals, Chile \\ A Calderón Antofagasta Minerals, Chile \\ R Castellón TIMining, Chile \\ M Vargas TIMining, Chile \\ C Mena TIMining, Chile \\ L Orellana TIMining, Chile \\ S Wiche TIMining, Chile \\ C Calderón TIMining, Chile
}

\begin{abstract}
Traditionally in open pit mining, predicting and sizing potential structurally controlled instabilities has been carried out by statistically processing field mapped structures and slope design parameters, mainly bench orientation and face angle. This methodology usually identifies plane faults (one structure) and wedges (two structures), whose estimated size and probability of existence are based on the assumption of the most unfavourable case. The above is a result of not having simple and fast tools that can provide a georeferenced prediction of instabilities, analysing real surfaces and not only simple benches, and identify polyhedral blocks (three or more structures) with their associated safety factor (SF) and assessing the stability of the blocks on a case-by-case basis.
\end{abstract}

This paper introduces the results of the implementation of algorithms that help to (1) make geolocalised prediction of structurally controlled instabilities using real and mine design surfaces, and to (2) calculate the SF for unstable blocks classified as either planar, wedge, or polyhedral failures, considering rotation. For this, examples from Chile's large-scale mining sites were used as study cases.

Doing a back-analysis exercise that was later proven in the constructed slope, plane failures, wedges and polyhedral blocks were detected in old design surfaces. In addition, potential instabilities were identified by extending the structures mapped at the current operation level to lower benches in the design surface, which were confirmed once those benches were built.

The safety factors calculated with this new methodology are consistent with the plane faults, wedges and blocks that were stable or unstable in the slopes evaluated. In the case of wedges, a sample of 207 wedges was evaluated obtaining that the $74 \%$ of cases with SF associated to this new methodology had less than 0.2 points of variation comparing to the SF calculated with other software of the industry. In the rest of the cases there is a relation between the bigger variation of SFs and the geometry of the wedges, where the biggest differences among the classic SF and this methodology is associated to the wedges that are more likely to rotate. In the case of polyhedral blocks, whose SF calculation had not been easily obtained with current methodologies, the SF obtained is consistent with those from representative wedges for these blocks.

By implementing these algorithms, identification and stability assessment of georeferenced blocks in a bench, wall, structural domain, and even an entire pit, based on actual onsite mapped structures, is streamlined. This enables operational and planning teams to predict geotechnical events and to promptly evaluate measures to improve operational continuity and safety of benches under construction.

Keywords: polyhedral blocks, instabilities, safety factor, structure, slope, rotation, real surface, prediction 


\section{Introduction}

Traditionally in open pit mining, predicting structurally controlled instabilities is done by statistically processing site mapped structures to identify structural sets or families. For each of these structural sets, the mean and standard deviation of their dip, dip direction, spacing and length are calculated to then simulate a spatial arrangement of these sets on a slope. This slope is usually generated in a linear fashion, with a userdefined length and parameters like slope angle and average berm width of the site.

The different software tools that apply this methodology simulate instabilities such as plane failures and wedges. As a result, we can identify which combination of structural sets forms each type of instability, allowing geotechnical engineers and geologists to prioritise the mapping of these sets.

When it comes to blocks formed by three or more structures, a methodology named 'Block Theory' (Goodman \& Shi 1985) has been established, which allows identification of blocks formed by any number of planes and with any geometry. These software solutions however also rely on analysing structural families and simulating slopes.

Analysing instability formation by using structural sets and slope simulation has been performed because up to now there were no automated tools capable of uploading a real topographic surface of a site or a design surface, and identifying instabilities in a geolocalised way, using field mapped structures and not only simulations of structures and topographic surfaces. In order to identify potential instabilities in a real slope, without a computer aid tool, it is necessary to identify the location of potential structures forming instabilities and to analyse if they have formed a block by a visual inspection, which requires a great amount of time and makes it difficult to analyse more than one zone at a time.

Several algorithms, which are described in this work, have been programmed today in a software called TIMining Tangram (TIMining 2019). These algorithms allow the user to upload real or design surfaces of a site, upload the mapped structures, and identify instabilities automatically. The software identifies plane failures, wedges, and also polyhedral blocks of three or more planes in real and design surfaces, thus predicting potential instabilities in the design surfaces.

Furthermore, the stability of the identified blocks is predicted by obtaining the safety factor (SF) for each block. The safety factor calculation has been studied and proposed by several authors for both plane and wedge failures (Chan \& Einstein 1981; Chen 2004; Hoek \& Bray 1981; Jiang et al. 2013; Jimenez-Rodriguez \& Sitar 2007; John 1968; Kumsar et al. 2000; Londe et al. 1969; Priest 1985; Wang \& Yin 2002; Wittke 1965; Yeung et al. 2003) as well as blocks made up of three or more structures (Goodman \& Shi 1985; Jiang \& Zhou 2017).

In the case of polyhedral blocks, the safety factor is generally obtained in the industry by applying the methodology defined by Goodman \& Shi (1985), however Jiang \& Zhou (2017) have examined the limitations of this theory and proposed a new methodology called the Rigorous Method.

This paper will present some improvements to the Rigorous Method of Jiang \& Zhou (2017) for the calculation of the safety factor of polyhedral blocks formed by three planes or more; will show the results of the implementation of these algorithms in TIMining Tangram and the results of the calculation of the safety factor of polyhedral blocks identified from real structures, in real and design topographic surfaces of large mining sites in Chile, owned by Antofagasta Minerals S.A. (AMSA). 


\section{$2 \quad$ Methodology}

\subsection{Identification and prediction of plane failures, wedges and polyhedral blocks on real surfaces of mining operations}

In order to identify and predict plane failures, wedges and polyhedral blocks using real mine site surfaces, the software features a series of algorithms. By means of these algorithms the users are able to:

1. Upload real and design topographic surfaces from a mine site visualising them as $3 \mathrm{D}$ triangle meshes.

2. Upload the database of site mapped structures, visualising by means of a georeferenced disk per structure. This database contains information of each structure like id, coordinates, dip, dip direction and length. The size of the disks that represent the structures will be the length mapped for each structure.

3. Upload the site mapped fault surfaces, visualising them as 3D triangle meshes.

4. Use these data to identify planar failures, wedges and blocks of three or more structural planes automatically and geolocalised in the real or design topographic surfaces of the mine site.

\subsubsection{Identification and prediction of plane failures}

To identify and predict plane failures, TIMining Tangram:

1. Allows you to enter a maximum angle difference between the slope strike and the structure strike for which a structure will be considered to be capable of originating a plane failure.

2. Generates the intersecting traces between all the structures and the topographical surface to be analysed, either real or design.

3. Evaluates which structures have an intersecting trace with a strike difference with the slope lower than or equal to the maximum angle entered to consider that a plane failure could be formed.

4. It generates a plane failure instability with each one of the structures that fulfill the preceding condition, the slope and a simulated liberating plane.

5. It stores each one of the identified plane failures, which are thus linked to the analysed surface and the structure that generates it.

\subsubsection{Identification and prediction of wedges and polyhedral blocks}

In order to identify and predict wedges and polyhedral blocks, TIMining Tangram:

1. Generates all intersections among topographic surface, structures and fault surfaces, including structures intersecting with other structures and fault surfaces intersecting with other fault surfaces.

2. Stores all structural planes and triangles of topographic meshes and fault surfaces involved at any intersection.

3. Analyses if these planes and triangles together with other planes and triangles stored form a closed volume.

4. If the closed volume is located beneath the topographic surface and is outcropping; it creates a block or wedge and stores it in a database.

5. If the closed volume is directly or indirectly associated with an outcropping block or wedge, it also creates a block or wedge and stores it in a database.

6. All blocks and wedges are associated with the analysed topographic surface and the structures or fault surfaces that form them. 


\subsection{Safety factor calculation}

\subsubsection{Classic safety factor}

In this paper, the term 'Classic Safety Factor' describes the safety factor that has been usually calculated in the industry, which considers only the forces acting on an instability but not the momentum influences acting on it. This is because it is assumed that the forces will pass through the centre of gravity of the instability, which omits the distance between the centre of gravity and the actual location of application of the force of each plane on the instability, and this in turn cancels out any momentum acting on the block (Goodman \& Shi 1985).

Furthermore, this Classic Safety Factor can only be calculated for plane failures and wedges, since the calculation of the normal forces acting on each plane, for blocks of three or more planes, using a system of equations generates a system with more unknowns than equations. In order to solve it, it is needed assumptions that distance the results from reality (Jiang \& Zhou 2017).

The Classic Safety Factor $\left(\mathrm{FS}_{\mathrm{c}}\right)$ is calculated by the following division (Hoek \& Bray 1981):

$$
\mathrm{FS}_{\mathrm{c}}=\text { resisting forces/sliding forces }
$$

where Resisting Forces are all the forces that counter the sliding forces causing instability, and Sliding Forces are those forces that facilitate it. In this paper, the Resisting Forces have been considered as the sum of the friction force and cohesion force, while the Sliding Forces have been considered as the weight in the sliding direction.

For plane failures:

$$
\mathrm{FS}_{\mathrm{c}}=(\mathrm{N} \tan (\varnothing)+\mathrm{CA}) / \mathrm{W}_{\mathrm{s}}
$$

where $N$ is the normal force of the plane on the instability, $\varnothing$ is the friction angle of the plane, $c$ the cohesion of the plane, $A$ the area of contact between the instability and the plane, and $W_{s}$ is the weight of the sliding block in the sliding direction.

In the case of wedges, if the wedge slides on a single plane, the safety factor is calculated in the same way as for a plane failure, and for this it has to be confirmed that there is only one plane with its normal vector pointing into the wedge. If the wedge slides on both planes, or in other words, on its axis, both normal vectors are pointing into the wedge and the safety factor is calculated as follows:

$$
F S_{c}=\left(N_{i} \tan \left(\varnothing_{i}\right)+N_{j} \tan \left(\varnothing_{j}\right)+c_{i} A_{i}+c_{j} A_{j}\right) / W_{s}
$$

where $\mathrm{i}$ and $\mathrm{j}$ are the structural planes that form the wedge.

\subsubsection{TIMining Tangram safety factor}

The new way of calculating the safety factor implemented in the TIMining Tangram software, where this calculation has been called simply safety factor, unlike the Classic Safety Factor, is based on the work done by Jiang \& Zhou (2017).

The authors developed a method called 'Rigorous Method' that calculates the safety factor for blocks consisting of multiple planes, considering both equilibrium of forces and momentum. To perform the calculation, the authors:

1. Divide the block into vertical columns, with as small a base as possible.

2. They develop a free body diagram or force diagram for each column, considering external forces, normal forces, the weight of each column, friction and cohesion forces and intercolumn forces.

3. They set the force and momentum equilibrium equations for each column:

$$
\sum_{\mathrm{l}=1}^{\mathrm{m}} \iint_{1} \sigma_{\mathrm{l}} \mathbf{v}_{\mathrm{l}} \mathrm{d} S+\sum_{\mathrm{l}=1}^{\mathrm{m}} \iint_{1} \tau_{\mathrm{l}} \mathbf{t}_{\mathrm{l}} \mathrm{d} S+\mathbf{f}=0
$$




$$
\sum_{\mathrm{l}=1}^{\mathrm{m}} \iint_{\mathrm{l}}\left(\mathbf{x} \times \mathbf{v}_{\mathrm{l}}\right) \sigma_{\mathrm{l}} \mathrm{dS}+\sum_{\mathrm{l}=1}^{\mathrm{m}} \iint_{\mathrm{l}}\left(\mathbf{x} \times \mathbf{t}_{\mathrm{l}}\right) \tau_{\mathrm{l}} \mathrm{d} S+\mathbf{m}=0
$$

where I ranges from 1 to $m$, represents each of the columns, $\sigma_{l} \mathbf{v}_{l} \mathrm{dS}$ is the normal force acting on the base of each column, $\tau_{1} t_{1} d S$ is the shear force acting on the base of each column, $\mathbf{f}$ is the resulting external force acting on the block, $\left(\mathbf{x} \times \mathbf{v}_{1}\right) \sigma_{1} \mathrm{dS}$ is the momentum induced by the shear forces in each column from its centre of gravity, $\left(\mathbf{x} \times \mathbf{t}_{1}\right) \tau_{1} \mathrm{dS}$ is the momentum induced by the shear forces in each column from its centre of gravity and $\mathbf{m}$ is the resulting external momentum acting on the block.

4. Using the safety factor formula stablished by the Mohr-Coulomb criteria:

$$
\mathrm{SF}=\left[\left(\sigma_{1}-\mathrm{u}\right) \tan \varnothing_{1}+\mathrm{c}_{1}\right] / \tau_{1}
$$

Where $u$ represents the pore pressure, $\sigma_{1}$ the normal stress on each column and $\tau_{1}$ the shear stress on each column; replace $\tau_{1}$ in Equations 4 and 5 , obtaining the equilibrium equations as a function of the safety factor:

$$
\begin{gathered}
\sum_{\mathrm{l}=1}^{\mathrm{m}} \iint_{\mathrm{l}}\left(\mathrm{SF} \mathbf{v}_{\mathrm{l}}+\tan \varnothing_{\mathrm{l}} \mathbf{t}_{\mathrm{l}}\right) \sigma_{\mathrm{l}} \mathrm{d} \mathrm{S}+\sum_{\mathrm{l}=1}^{\mathrm{m}} \iint_{\mathrm{l}} \mathbf{t}_{\mathrm{l}}\left(\mathrm{c}_{\mathrm{l}}-\mathrm{utan} \varnothing_{\mathrm{l}}\right) \mathrm{d} \mathrm{S}+\mathrm{SF} \mathbf{f}=0 \\
\sum_{\mathrm{l}=1}^{\mathrm{m}} \iint_{1}\left[\mathrm{SF}\left(\mathbf{x} \times \mathbf{v}_{\mathrm{l}}\right)+\tan \varnothing_{\mathrm{l}}\left(\mathbf{x} \times \mathbf{t}_{\mathrm{l}}\right)\right] \sigma_{\mathrm{l}} \mathrm{d} \mathrm{S}+\sum_{\mathrm{l}=1}^{\mathrm{m}} \iint_{\mathrm{l}}\left(\mathbf{x} \times \mathbf{t}_{\mathrm{l}}\right)\left(\mathrm{c}_{\mathrm{l}}-\mathrm{utan} \varnothing_{\mathrm{l}}\right) \mathrm{d} \mathrm{S}+\mathrm{SFm}=0
\end{gathered}
$$

5. The Equations 7 and 8 are written in a matrix form to obtain a single equation, which is solved by iterative methods until it converges to a solution, thus finding the safety factor.

In this work, slight modifications and improvements have been made to Jiang \& Zhou's work (2017), which are as follows:

1. The algorithm was identified as having difficulty converging when the slip angle (Jiang \& Zhou 2017) tends to $\pm 45^{\circ}$ and $\pm 135^{\circ}$, angles that coincide with the asymptotes $x=y$ or $x=-y$ that has the polynomial function that models the normal internal forces (Jiang \& Zhou 2017). Therefore, the following polynomial approximation for internal normal forces was used for each column: $\Lambda_{\mathrm{i}}=$ $\left(\lambda_{1} \lambda_{2} x_{i}^{2} \lambda_{3} y_{i}^{2} \lambda_{4} x_{i} y_{i}\right)^{\top}$. This resulted in a large number of previously divergent cases converging when the slip angle tended to $\pm 45^{\circ}$ and $\pm 135^{\circ}$.

2. The algorithm was identified as sensitive to the translation of the coordinate system, affecting the calculation of the momentums. For this, the coordinate system was centred on the centre of gravity of each block.

3. The reason for the slow divergence or convergence of the algorithm was identified as being that there are two solutions for the safety factor, one of which has always been invalid so far, since it is negative. Both solutions have opposite slip vectors (they differ by $180^{\circ}$ ). For this:

a. The slip angle change velocity was restricted in the iterative process to approximately $20^{\circ}$ per iteration, which improved convergence and reduced convergence cases to a negative safety factor.

b. A slip angle estimate was made based on the normal vectors of the planes forming the block. These normal vectors were averaged and weighted by the contact area of each plane, considering only the normal vectors with a negative vertical component (makes it easier for the block to fall). When the convergence of the iterative method resulted in a negative safety factor, an initial value of the slip angle equal to the aforementioned estimation was used, plus $180^{\circ}$. This also reduced the cases of convergence to a negative safety factor.

4. Since the Jiang \& Zhou (2017) algorithm considers that there is no topographic surface or other block on top the block, i.e. the upper part of each of the columns is adjacent to air, the safety factor implemented by TIMining Tangram for blocks that are not $100 \%$ free shows the result as if they are. In these cases, the safety factor is such that it reflects the worst case in which the blocks or the part of the topographic surface that prevented them from being fully removable were already detached from the slope. 
5. Given that the Jiang \& Zhou (2017) algorithm is only applicable to blocks with certain geometries in such a way that their representative columns do not touch the topographic surface or the outer part of the block twice, a $1 \%$ tolerance was given so that it can consider blocks with very irregular shapes and obtain a safety factor estimation.

\subsection{Safety factor validation}

In order to validate the safety factor calculation implemented in TIMining Tangram, 207 wedge cases were created in the software and their safety factor was calculated in TIMining Tangram and Swedge (Rocscience Ltd. 2001) in order to compare them and find out the extent of deviation between the two. This case includes some plane failures due to the dip of one of the structures, when they act as liberating planes.

Since TIMining Tangram considers force and momentum equilibrium in the safety factor calculation and Swedge only considers force equilibrium, the safety factors of both software tools are not expected to be equal but similar in most cases.

In the remaining cases, the difference between the safety factors is expected to be associated with the inclusion of the momentum equilibrium.

\section{Data}

\subsection{Case 1: case study from 'Mine Site 1' of Antofagasta Minerals, Chile}

In this case study, an old design surface from one of the pits of the mine site was used to assess whether TIMining Tangram could find the instabilities that had eventually formed on the slope. The instabilities that formed are shown in Figure 1.

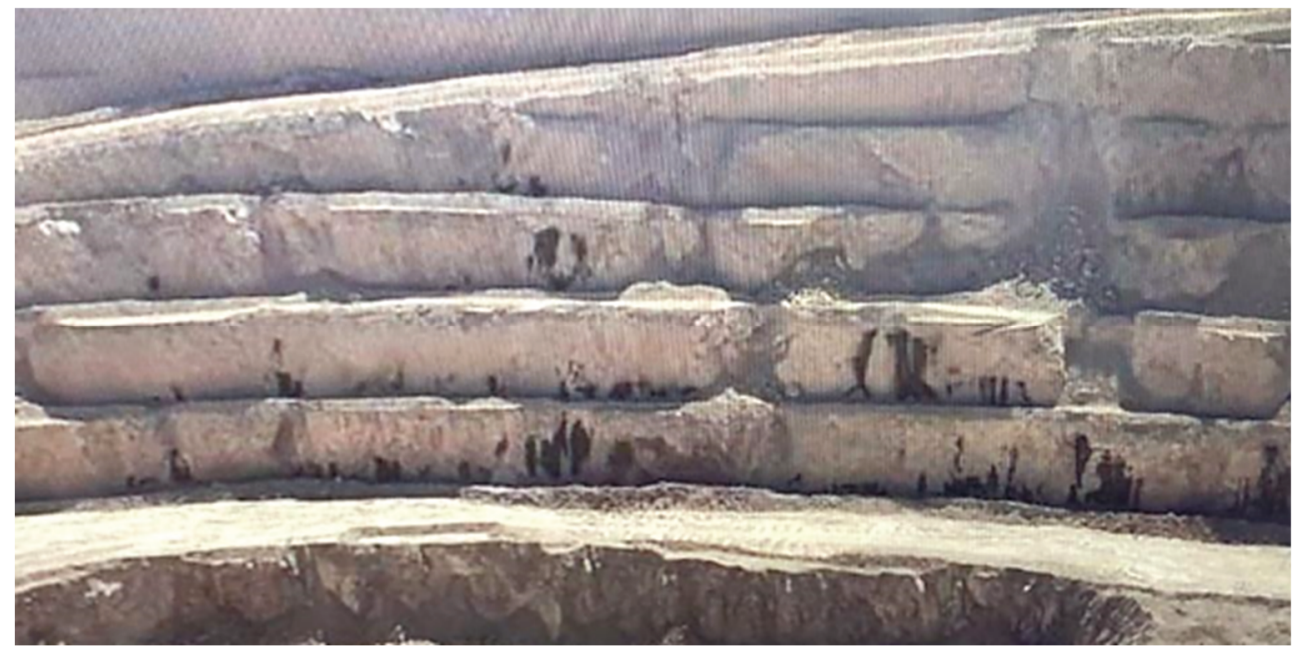

Figure 1 Instabilities formed in one of the pits of this mine site

To this end, the structures that formed these instabilities were mapped in the old design surface using the software in a georeferenced way. These structures are shown in Figure 2. 


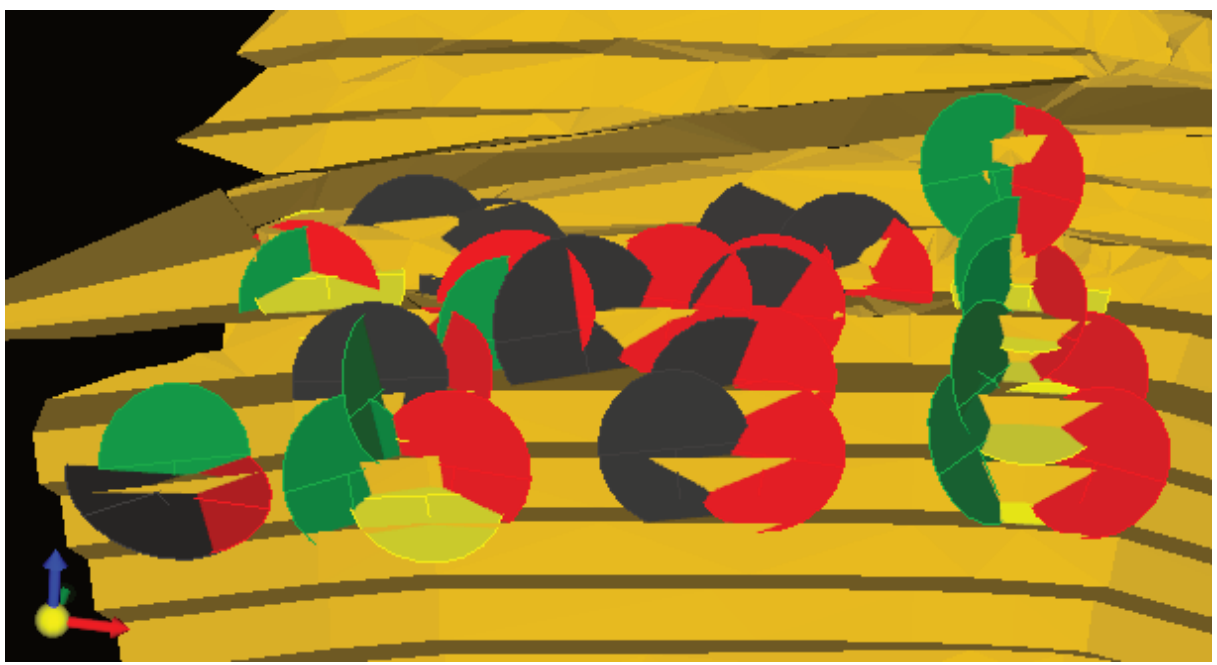

Figure 2 An old design topographic surface of this mine site, where the structures generating instabilities in the slopes were mapped, once built

\subsection{Case 2: case study from 'Mine Site 2' of Antofagasta Minerals, Chile}

TIMining Tangram was used in this case study to evaluate if a design surface proposed by the Planning Superintendence of this mine site for a determined zone was safe enough, due to some instabilities that were formed in the upper benches. These instabilities fell and were contained by the lower berm, but they filled almost all the berm, leaving it without capacity of contain other instabilities.

The mine site, in this zone, has a double bench surface, so given the previous case of instabilities fall, it is necessary to evaluate if the berm of the double bench is going to contain the rocks if they fall or if it is necessary to change the design to a simple bench in this phase.

The initial design surface and the structures to analyse are shown in Figure 3. These structures were mapped on the real surface of the mine, specifically on the last bench built. Then, these structures were projected to the design surface to analyse the next benches.

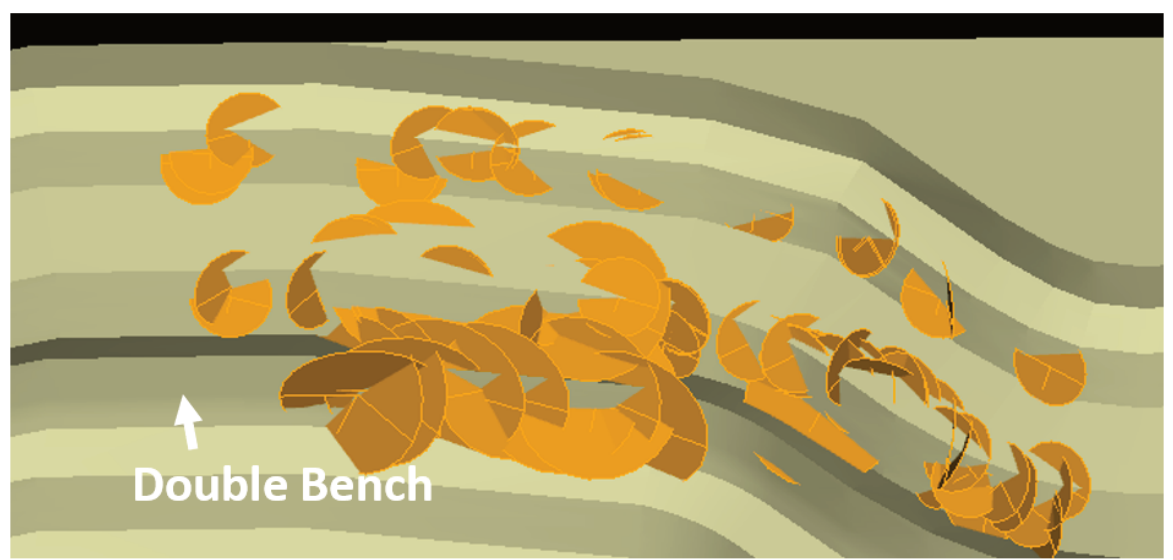

Figure 3 A design surface proposed by the Planning Superintendence of the mine site and structures to be analysed with

\subsection{Case 3: safety factor comparison between wedges identified with TIMining Tangram and similar wedges calculated in Swedge}

207 wedges were generated in TIMining Tangram and Swedge, on a $15 \mathrm{~m}$ high slope, with $70^{\circ}$ bench face angle and $20 \mathrm{~m}$ berm width. 
Of the 207 cases, 72 were generated by varying each wedge parameter such as dip, dip direction, cohesion and friction angle one by one. The rest of the cases were generated intersecting four structural sets with the slope. These sets have the following dip and dip direction: 30/180, 70/201, 36/93 and 60/240, each having a standard deviation of $10^{\circ}$ in dip and dip direction.

\section{$4 \quad$ Results}

\subsection{Case 1: case study from 'Mine Site 1' of Antofagasta Minerals, Chile}

Once plane failures, wedges and blocks of three or more planes were identified, rock blocks that effectively formed in the real topographic surface were also identified, which are shown in light blue in Figure 4.

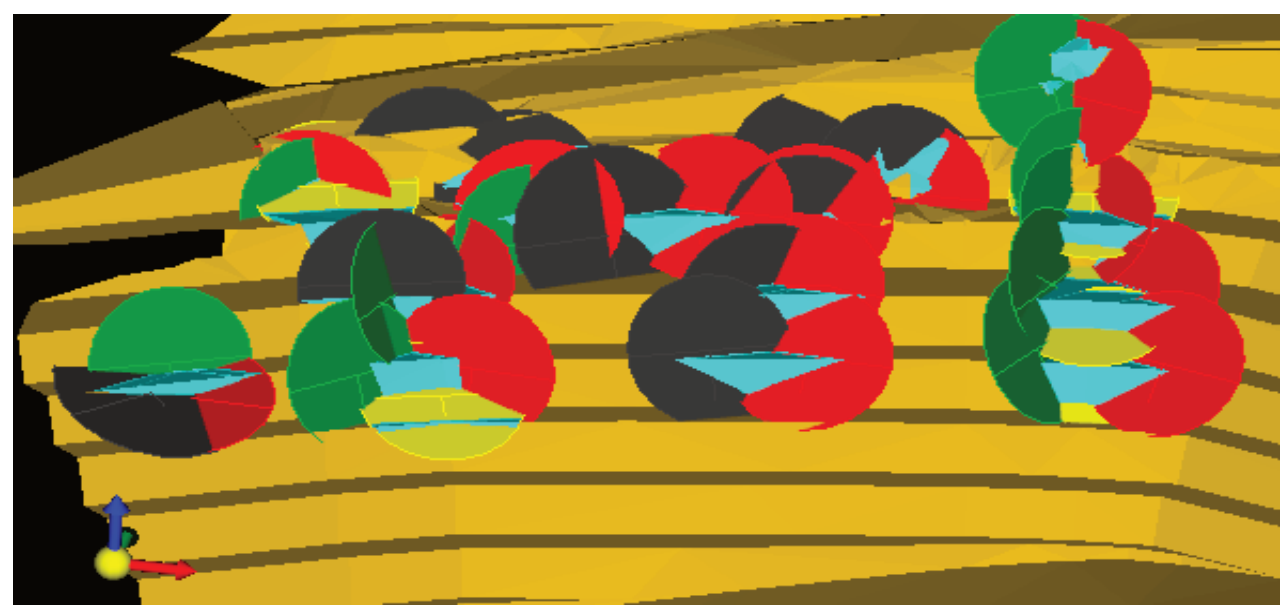

Figure 4 Instabilities such as plane failures, wedges and polyhedral blocks that actually formed in the real topographic surface of the mine site

The safety factor was calculated for the rock blocks that were identified. This allowed us to visualise which blocks could actually fail, that they have a safety factor lower than 1 (Figure 5).

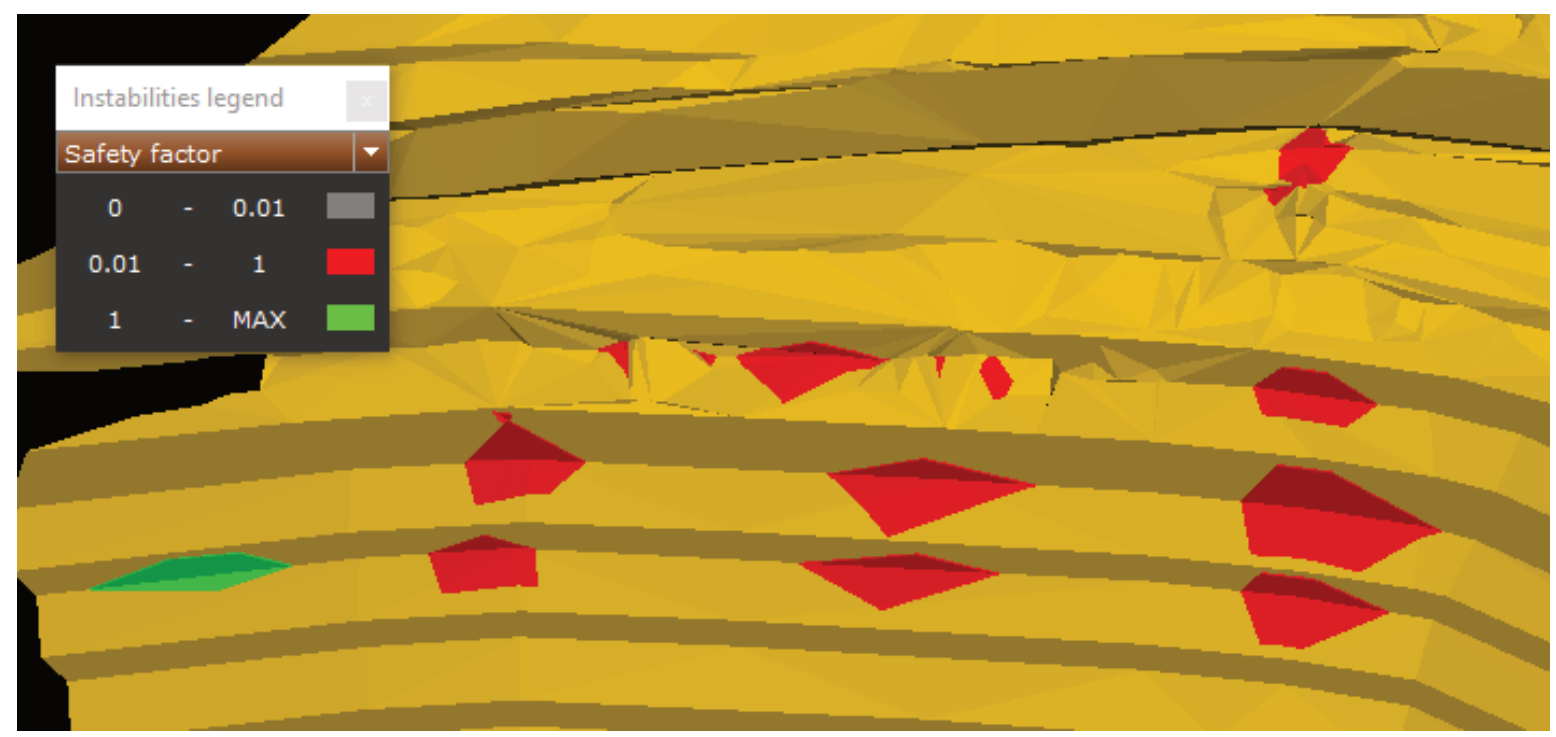

Figure 5 Coloured instabilities by safety factor (SF); red: SF lower than 1, green: SF higher than 1.2

Finally, rock blocks with a safety factor lower than 1 were removed from the topographic surface, with a resulting topographic surface shown on the left in Figure 6, and the real instabilities formed on the right in Figure 6. 

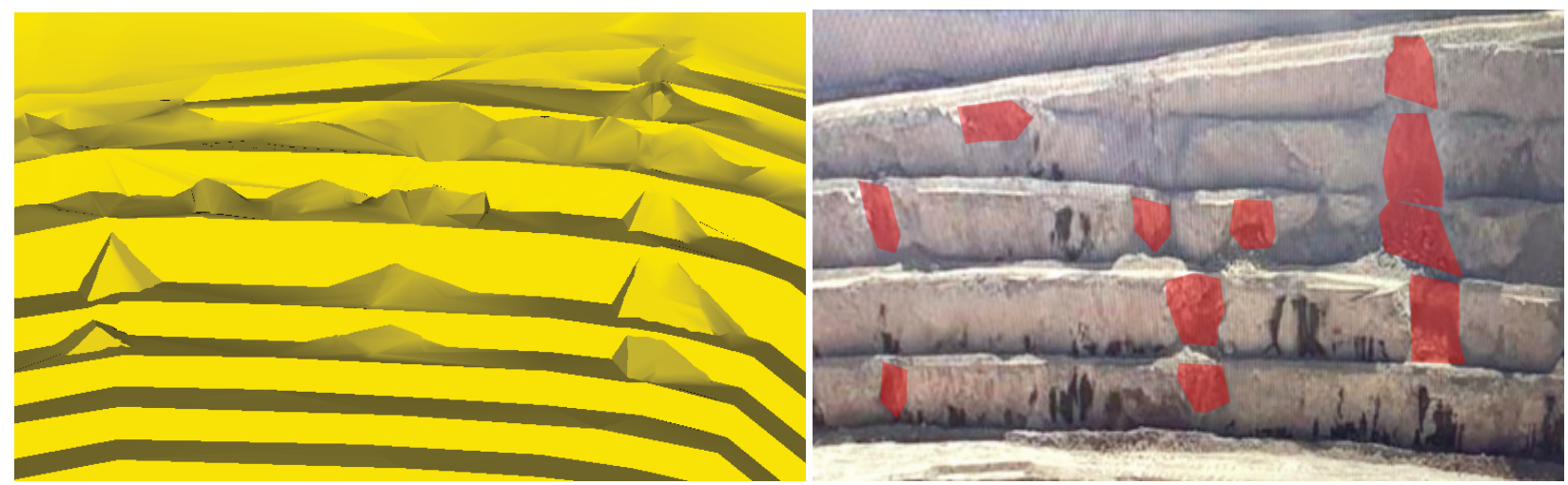

Figure 6 Left: Unstable blocks removed from the surface in TIMining Tangram. Right: unstable blocks that have already fallen or were removed from the surface in the mine site

This exercise demonstrates that TIMining Tangram was able to identify the nine rock blocks simulated and that there were formed in the mine, and calculate as in the reality, that these blocks had a safety factor lower than 1. This means that by loading the site mapped structures in TIMining Tangram, you can identify instabilities that have not yet fallen in the real topographic surface as well as potential unstable blocks in design surfaces, calculating their safety factor and obtaining a topographic surface that simulates how the slope would look once the unstable blocks have fallen or have been removed.

\subsection{Case 2: case study from 'Mine Site 2' of Antofagasta Minerals, Chile}

In this case, 17 rock blocks were detected on the initial design surface of the mine site, as shown in Figure 7. The volume of them varies from $150 \mathrm{~m}^{3}$ to $10,800 \mathrm{~m}^{3}$ approximately. The Figure 7 shows the biggest blocks number 1,2 and 3 , whose volume are $10,800 \mathrm{~m}^{3}, 3,350 \mathrm{~m}^{3}$ and $4,530 \mathrm{~m}^{3}$ respectively.

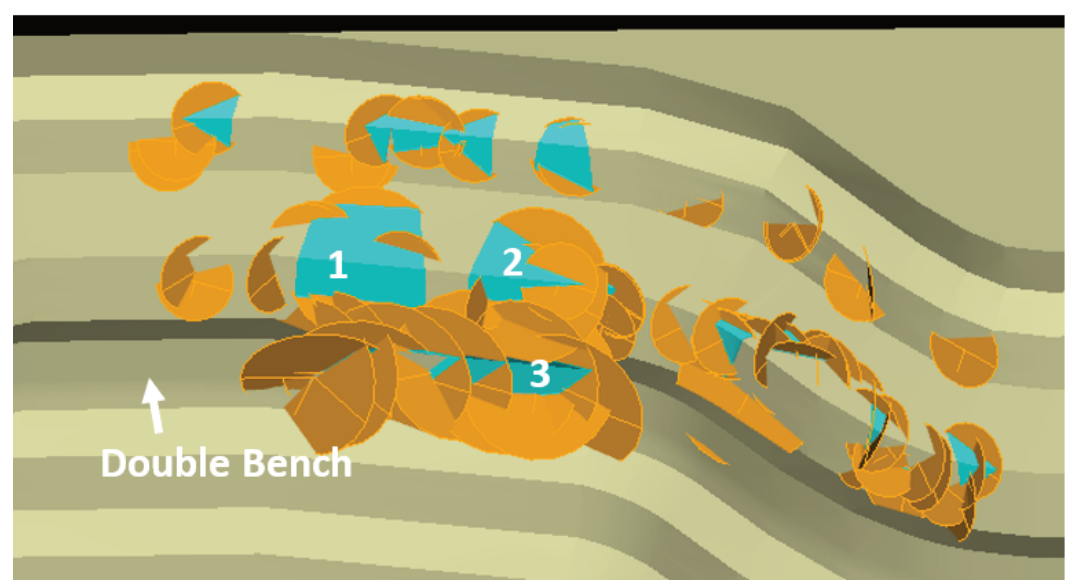

Figure 7 Instabilities detected on initial design surface of the mine site, where numbers 1,2 and 3 are the biggest ones

Then the safety factor was calculated (Figure 8), obtaining that blocks numbered 1 and 3 had safety factors within the safety margin of the mine, which is between 1 and 1.2 values. 


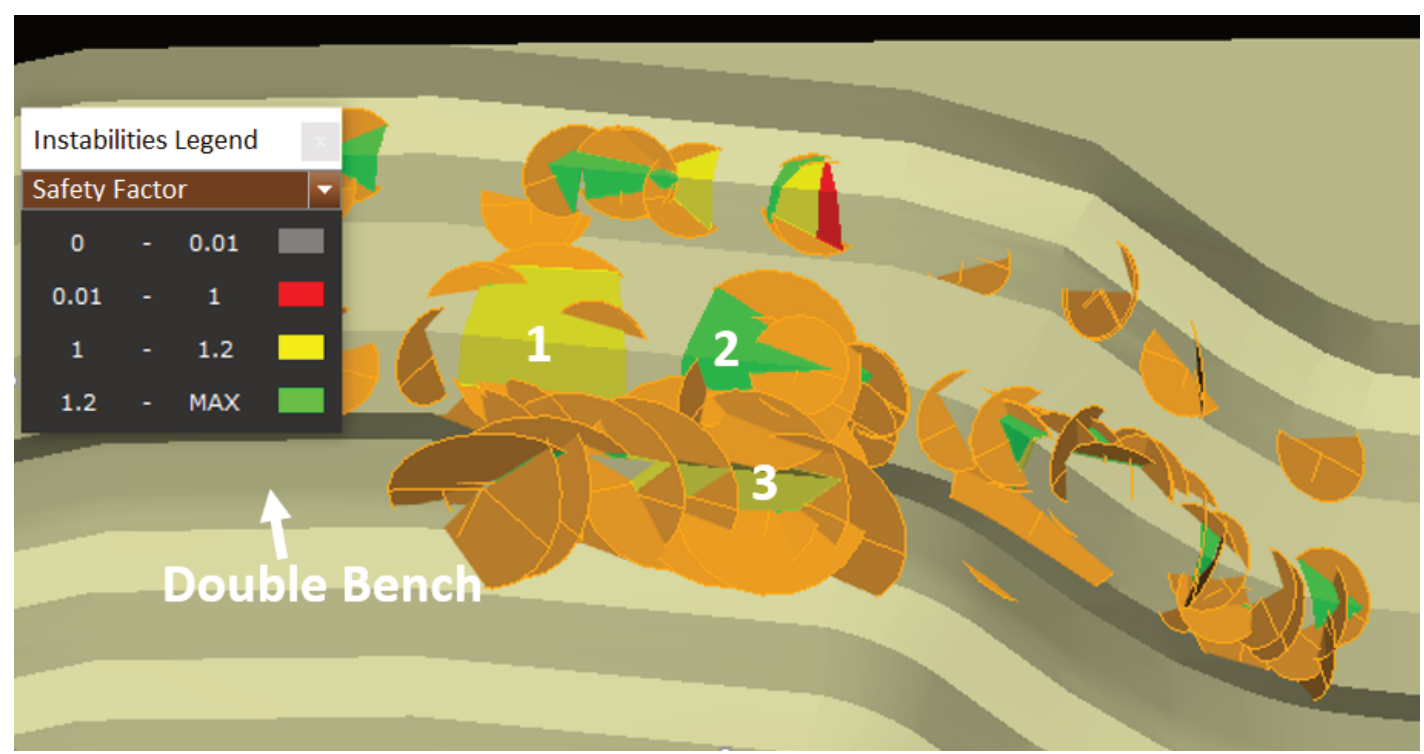

Figure 8 Safety Factors of each instability detected in an initial design topography of the mine site, where instabilities number 1 and 3 have safety factors under 1.2, limit of the safety margin of the mine

As a result of the detection of instabilities and safety factor calculation, the geotechnical area proposed the Planning Superintendent for safety reasons to conduct a redesign, to reduce the probability of having a geotechnical event and to have berms that could contain the blocks, given that the berms of the original design are too narrow to contain this amount of fallen volume. This resulted in a design change from a double bench to a simple bench shown in Figure 9, with the aim that it contains instabilities if they eventually fall.

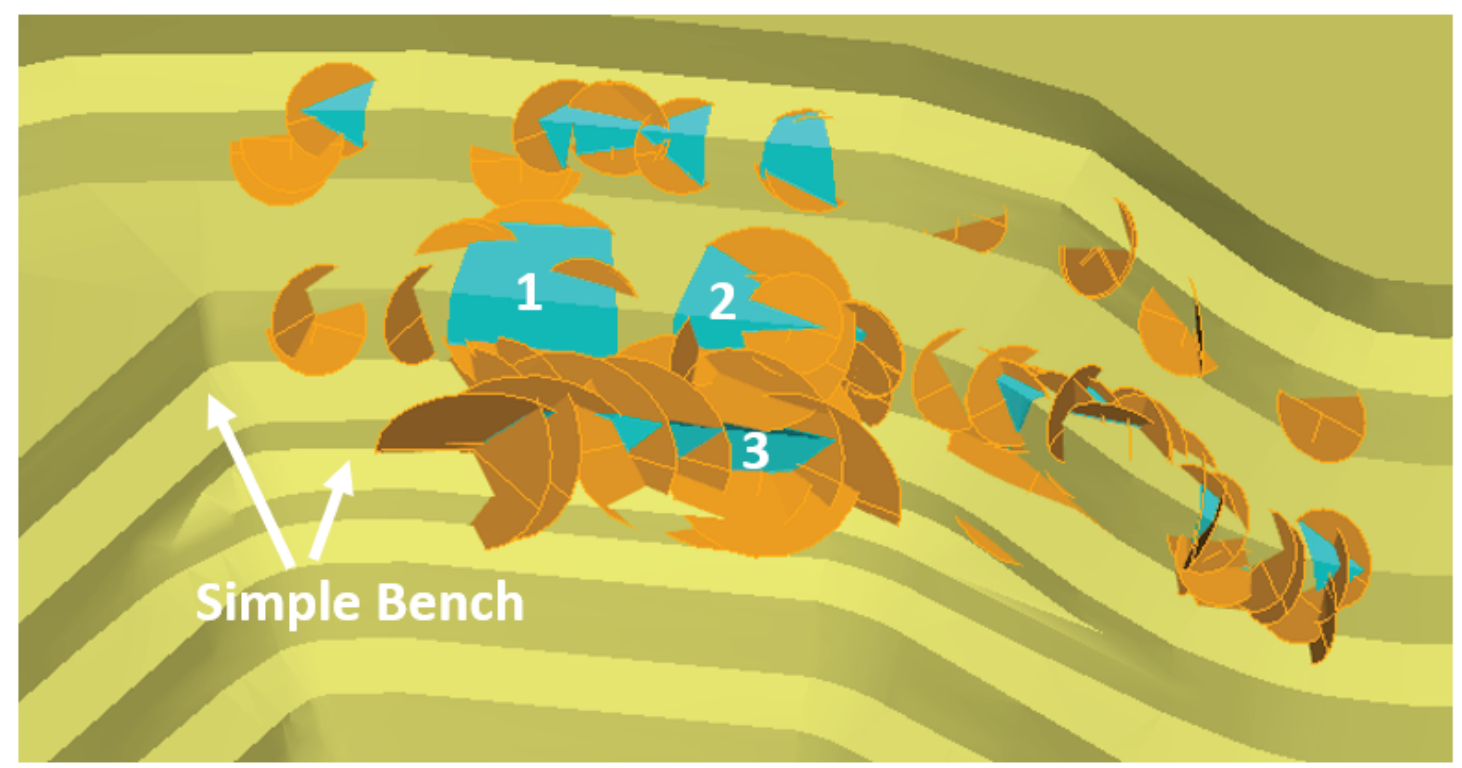

Figure 9 New design surface proposed by the Planning Superintendent, which is formed by simple benches to contain the instabilities that could eventually fall

\subsection{Case 3: safety factor comparison between wedges identified with TIMining Tangram and similar wedges calculated in Swedge}

By calculating TIMining Tangram and Swedge safety factors for the 207 wedge cases, the ratio shown in Figure 10 was obtained: 


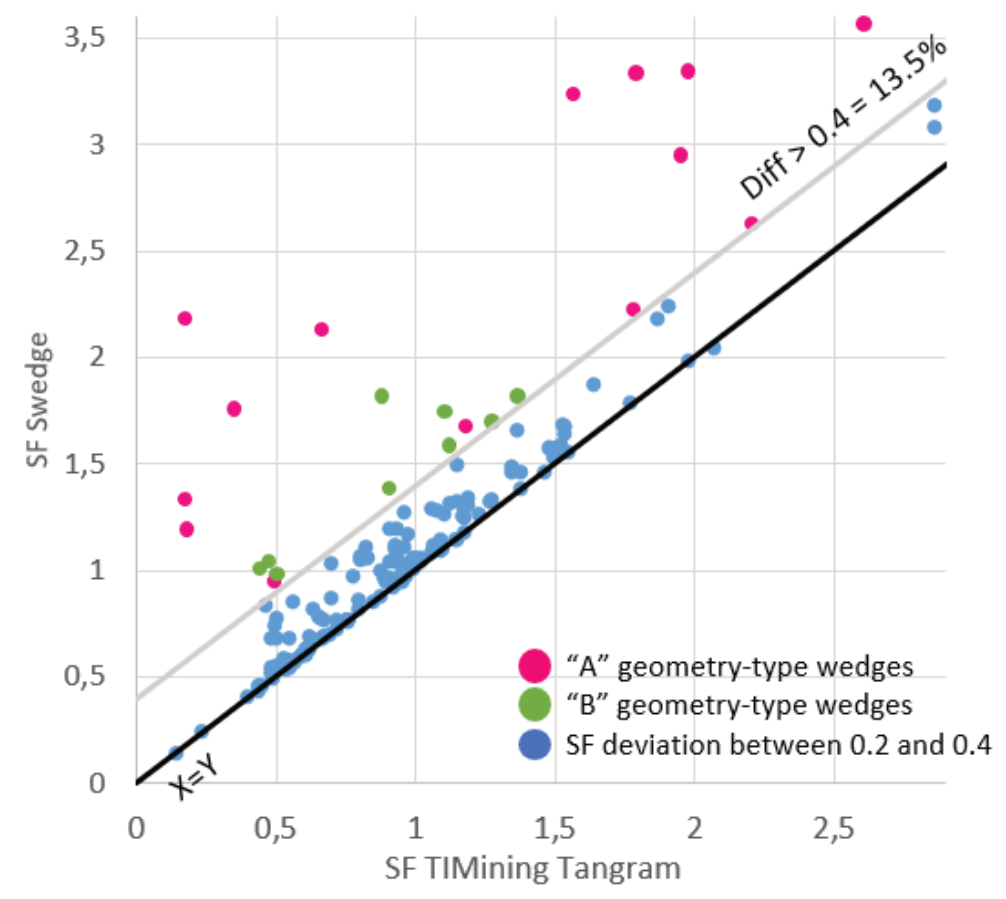

Figure 10 Ratio between TIMining Tangram and Swedge safety factor for 207 wedge cases. $74 \%$ of the cases present a difference of less than $0.2 ; 11.6 \%$ of the cases a difference between 0.2 and 0.4 , and $13.5 \%$ of the cases a difference greater than 0.4 . In $1 \%$ of the cases the TIMining Tangram safety factor did not converge. See ' $A$ ' and 'B' geometry-type wedges in Figure 11

The data in Figure 10 show that in $74 \%$ of the cases, the difference between the two safety factors was less than 0.2 , in $11.6 \%$ of the cases, there was a difference between 0.2 and 0.4 , and in $13.5 \%$ of the cases, there was a difference greater than 0.4 . In $1 \%$ of the cases, TIMining Tangram's safety factor did not converge.

When the difference was greater than 0.4 , the wedge geometry was analysed, thus finding that there were two large groups of distinct geometries. The cases shown in Figure 10 in pink account for $4.8 \%$ of the total cases and they are wedges of ' $A$ ' type geometries (Figure 11). The cases shown in Figure 10 in green account for $6.8 \%$ of the total number of cases and are wedges of ' $B$ ' type geometries (Figure 11).

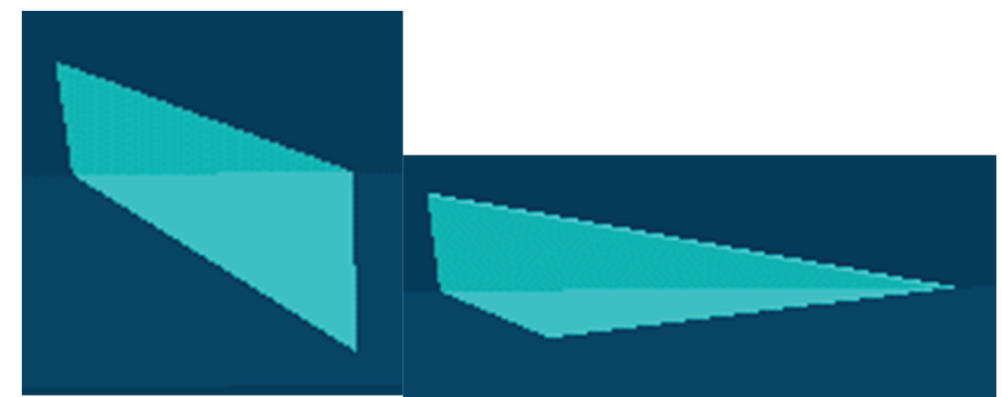

(a)

(b)

Figure 11 Of the total number of cases, $4.8 \%$ reported a difference between both safety factors greater than 0.4 , and have ' $A$ ' type wedge geometry, which presents a dip close to $90^{\circ}$ in one of its structures. $6.8 \%$ of all cases presented a difference greater than 0.4 between both safety factors, and have ' $\mathrm{B}$ ' type wedge geometry, which is a horizontal needle-shaped wedge with a contact area in one of its structures that is much larger than the other

Since these ' $A$ ' type wedges have a structure with a dip close to $90^{\circ}$, when dividing the block into columns, the number of columns that remain in contact and therefore represent the forces associated with this structure is much less than the number of columns that remain in contact with the other structure. This 
means a poor representation of the forces associated with the structure with a dip close to $90^{\circ}$, hence there is an opportunity to improve the safety factor calculation for this type of wedge.

For ' $B$ ' type wedges, the difference between the two safety factors reflects the consideration of the momentum equilibrium in TIMining Tangram's safety factor calculation, since most of the wedge's tonnage rests on the structure with the largest contact area, with the wedge axis acting as a pivot, and a tendency to rotate as it falls.

\section{Conclusion}

The results showed in this paper indicates that with the new methodology implemented in TIMining Tangram software, it is possible to identify and predict geolocalised instabilities using real surfaces and structures mapped in a mine operation. The instabilities detected are not only formed by one or two structural planes, but by three or more structural planes, with any geometry. Also, the new methodology implemented to calculate the safety factor allows to know whether a block formed by n-structural planes is stable or not, which hadn't been possible until now considering all the forces and momentums acting in every plane of the instability.

For this reason, this tool has been implemented in all the great mining operations of Antofagasta Minerals in Chile: Minera Antucoya, Minera Centinela, Minera Los Pelambres and Minera Zaldívar; used as a corporate standard by geologists and geotechnical engineers.

This advance allows improvement in the safety and the operational continuity at the mine site, because now the geomechanical team is able to predict the stability of blocks with any shape and take preventive actions to minimise the fall of blocks that generates delays in the mining plan and generates accidents. Also, it is possible to analyse many design surfaces together with their potential berm losses, creating design surfaces safer.

The 'Mine Site 2' case study shows how this tool could be used to predict instabilities in design surface for the aim to avoid accidents and improve the operational continuity, where a new design surface was proposed by the Planning Superintendence to better contain potential instabilities if they fall.

The 'Mine Site 1' case study shows that TIMining Tangram actually detected the instabilities that formed on the slope. Subsequently, when calculating the safety factor, blocks with a safety factor lower than 1 obtained a result that is consistent with those obtained in the actual surface, where these blocks actually fell.

The safety factor calculation for the 207 wedge cases, compared to Swedge's safety factor, has also yielded consistent results. $74 \%$ of the cases present less variation than 0.2 points with respect to the Swedge safety factor, which are the cases in which the momentum values do not play a significant role in block stability. For all other cases, there is an important group of wedges where the variation between the safety factors is explained by the action of the momentum values on the block. Finally, there is an opportunity to improve the algorithm implemented for wedges with structures with a dip close to $90^{\circ}$, and for those cases in which the assumption was that the blocks did not have other blocks or part of the topographic surface above them, where the worst case safety factor is obtained, which is that these blocks are not blocked by other blocks or the topographic surface.

On this last point, it is worth noting the case of embedded blocks. These blocks will have a very low safety factor because the algorithm will assume that the surface in front of them does not exist, and therefore tend to overturn. Thus, further analysis is required in these cases.

\section{Acknowledgement}

This effort was carried out thanks to an innovation project between Antofagasta Minerals S.A. (AMSA) and TIMining, co-financed by Innovaminerals (AMSA innovation area) and TIMining, promoted by Alex Calderón (AMSA) and led by Felipe González (AMSA). Felipe González also provided the data to present the Mine Site 1 case study, as did Alex Tabilo for the Mine Site 2 case study. 


\section{References}

Chan, H \& Einstein, H 1981, 'Approach to complete limit equilibrium analysis for rock wedges - the method of 'artificial supports", Rock Mechanics, vol. 14, no. 2, pp. 59-86.

Chen, Z 2004, 'A generalized solution for tetrahedral rock wedge stability analysis', International Journal of Rock Mechanics and Mining Sciences, vol. 41, pp. 613-628.

Goodman, R \& Shi, G 1985, Block Theory and its Application to Rock Engineering, Prentice-Hall, London.

Hoek, E \& Bray, J 1981, Rock Slope Engineering, 3rd edn, Institute of Minerals and Metallurgy, London.

Jiang, Q, Liu, X, Wei, W \& Zhou, C 2013, 'A new method for analyzing the stability of rock wedges', International Journal of Rock Mechanics and Mining Sciences, vol. 60, pp. 413-422.

Jiang, Q \& Zhou, C 2017, 'A rigorous solution for the stability of polyhedral rock blocks', Computers and Geotechnics, vol. 90, pp. 190-201.

Jimenez-Rodriguez, R \& Sitar, N 2007, 'Rock wedge stability analysis using system reliability methods', Rock Mechanics and Rock Engineering, vol. 40, no. 4, pp. 419-427.

John, K 1968, 'Graphical stability analysis of slopes in jointed rock', Journal of the Soil Mechanics and Foundations Division, vol. 94, no. 2, pp. 497-256.

Kumsar, H, Aydan, I \& Ulusay, R 2000, 'Dynamic and static stability assessment of rock slopes against wedge failures', Rock Mechanics and Rock Engineering, vol. 33, no. 1, pp. 31-51.

Londe, P, Vigier, G \& Vormeringer, R 1969, 'Stability of rock slopes, a three-dimensional study', Journal of the Soil Mechanics and Foundations Division, vol. 95, no. 1, pp. 235-262.

Priest, $\mathrm{S}$ 1985, Hemispherical projection methods in rock mechanics, London.

Rocscience Ltd. 2001, Swedge - Probabilistic Analysis of the Geometry and Stability of Surface Wedges, computer software, Toronto, Canada, www.rocscience.com

TIMining 2019, TIMining Tangram - Software for Detection of Instabilities with Structural Control, computer software, Santiago, www.timining.com

Wang, Y \& Yin, J 2002, 'Wedge stability analysis considering dilatancy of discontinuities', Rock Mechanics and Rock Engineering, vol. 35, no. 2, pp. 127-137.

Wittke, W 1965, 'Method to analyse the stability of rock slopes with and without additional loading' (in German), Felsmechanik und Ingenieurgeologie, vol. 30, no. 2, pp. 52-79.

Yeung, R, Jiang, Q \& Sun, N 2003, 'Validation of block theory and three dimensional discontinuous deformation analysis as wedge stability analysis methods', International Journal of Rock Mechanics and Mining Sciences, vol. 40, pp. 265-275. 
Automated geolocalised identification of polyhedral blocks and their safety factor calculation in open pit mining 